\title{
Induced ferroelectric phase transformation in polycrystalline $\mathrm{BaTiO}_{3}$
}

\section{Lemos da Silva and M. Hinterstein}

\author{
Institute for Applied Materials, Karlsruhe Institute of Technology, 76131 Karlsruhe, Germany
}

lucas.silva@kit.edu

Electronic devices are becoming more and more present in our daily life and their relevance will increase even more in the future. These gadgets evolve aiming at higher performance, lower energy consumption and better portability. This continuously creates a demand for miniaturised components. Such devices contain many elements whose properties are based on ferroelectricity. Barium titanate is the model ferroelectric system. In addition, its properties are highly temperature and grain size dependent. It has excellent properties with grain sizes of approximately $1 \mu \mathrm{m}$ but undergoes marked weakening as the grain size decreases. A wide range of unimodal grain size distribution between $0.4 \mu \mathrm{m}$ and $15 \mu \mathrm{m}$ was successfully sintered, avoiding abnormal grain growth. Samples with intermediate grain size, showed excellent electromechanical and dielectric properties. They possess a balance between microstructural strain, existence and mobility of domain walls, which in turn allows the field induced crystal phase transformation. The samples, under application of external electric field, were subjected to in situ high energy X-ray diffraction analysis. To resolve the angular resolution a high-resolution multianalyser detector was used. By means of STRAP the field induced phase transformations were quantified. This induced phase transformation is stronger in samples whose grain size distribution curve is located around $1 \mu \mathrm{m}$. These results contribute to the understanding of fundamental questions about the ferroelectric effect in barium titanate and consequently other similar systems.

Keywords: barium titanate; induced phase transformation; grain size.

The authors would like to thank the Deutscher Akademischer Austauschdienst (DAAD, Bonn, Germany) for scholarship funding and the Deutsche Forschungsgemeinschaft (DFG) for funding. We acknowledge DESY (Hamburg, Germany), a member of the Helmholtz Association HGF, for the provision of experimental facilities. Parts of this research were carried out at beamline P02.1. 